\title{
Um Modelo de Treinamento para Programas de Melhoria de Processo de Software
}

\author{
Christiane Gresse von Wangenheim ${ }^{1,2}$, Jean Carlo Rossa Hauck ${ }^{1,2}$, \\ Aldo von Wangenheim ${ }^{1}$ \\ ${ }^{1}$ Universidade Federal de Santa Catarina - Florianópolis - SC - Brasil. \\ ${ }^{2}$ Universidade do Vale do Itajaí - UNIVALI - LQPS - Laboratório de Qualidade e \\ Produtividade de Software, Campus VII - São José - SC - Brasil \\ gresse@gmail.com, jeanhauck@egc.ufsc.br, awangenh@inf.ufsc.br
}

\begin{abstract}
An important factor for successful software process improvement are people, who need to be motivated and adequately trained to perform the process(es) being improved. Yet, software process improvement approaches, generally, do not provide detailed information on when to train what to which level of detail. Therefore, in this paper, we describe an iterative and incremental training approach for SPI initiatives, which has been developed based on ISO 10015 and practical SPI experiences. We also report experiences and lessons learned on applying the approach in a software process improvement initiative at the CYCLOPS Group/ Federal University of Santa Catarina/Brazil.
\end{abstract}

Resumo. Um dos fatores mais importantes para o sucesso de programas de melhoria de processo de software são as pessoas, que precisam ser treinadas e motivadas para a melhoria. Atualmente as abordagens de melhoria de processo de software, em geral, não fornecem subsidios detalhados de como e em que nivel treinar as pessoas em um programa de melhoria. Neste artigo,é descrita uma abordagem iterativa e incremental para iniciativas de SPI, que foi desenvolvida com base na norma ISO 10015 e em experiências práticas. Também são relatadas as experiências e lições aprendidas na aplicação da abordagem em uma iniciativa de melhoria de processo de software no grupo de pesquisa CYCLOPS da Universidade Federal de Santa Catarina/Brasil.

\section{Introdução}

Recursos humanos diretamente envolvidos em programas de melhoria de processo de software são fator determinante no seu sucesso. Pessoas necessitam ser motivadas e precisam de suporte para desenvolver as capacidades necessárias para executar os processos que estão sendo foco da melhoria. Entretanto estes participantes podem apresentar dificuldades em áreas específicas do conhecimento envolvido para executar o processo, como, por exemplo, na gerência de projetos. Muitas vezes este gap de conhecimento é decorrente de treinamento inapropriado [McCahon 96] [Wiegers 96]. Detectar e resolver dificuldades encontradas pela equipe envolvida na melhoria de processo é crucial. 
Micro e pequenas empresas (MPEs), em especial, sentem dificuldades em implantar melhoria de processos [Richardson e Wangenheim 07] [MCT 05] [SEBRAESP 00]. Apesar da existência dos diversos modelos de referência para a melhoria de processos de software disponíveis atualmente [SEI 06] [ISO/IEC 06] [SOFTEX 06], muitas MPEs simplesmente desconhecem ou não sabem aplicar corretamente estes modelos de referência [Storey 82] [Daft 92] [Johnson 99]. Acredita-se, que muito deste desconhecimento dos modelos pode ser decorrente da ineficácia dos modelos de treinamento disponíveis.

Tem-se observado na prática que treinamentos aplicados nos esforços de melhoria, em geral, não têm sido alinhados à metodologia utilizada no processo de melhoria em si e, portanto, não atendem às metas do programa de melhoria. Para que iniciativas de melhoria de processo de software tenham resultados positivos, é de grande importância que o treinamento esteja adequadamente alinhado aos objetivos da melhoria [McCahon 96].

Experiências têm demonstrado que as iniciativas de treinamento não têm atendido os objetivos da melhoria, porque muitas vezes possuem um enfoque por demais teórico, ou quando abrangem a prática dos processos, não se baseiam em exemplos concretos da organização, ou pelo menos, do domínio de aplicação de cada organização específica que sejam familiares aos participantes. Isto tem sido evidente em iniciativas de melhoria de processo colaborativas, envolvendo diversas MPEs em conjunto. Em iniciativas deste tipo, o treinamento é tipicamente ministrado em blocos de conteúdo, normalmente organizados por processo, que são voltados a um dos processos a serem aplicados na melhoria, reunindo as organizações envolvidas em uma única unidade de treinamento [Varkoi e Mäkinen 99] [Thiry et al. 07]. Mas durante um programa de melhoria, dependendo da abordagem de melhoria a ser seguida, e do modelo de referência de processo a ser adotado (CMMI-DEV [SEI 06], MPS.BR [SOFTEX 07], ISO/IEC 15504 [ISO 06], etc.), existem diferentes necessidades de conhecimento para cada etapa da iniciativa de melhoria e também para os diferentes papéis envolvidos.

Tendo identificado estas necessidades, observamos, que basicamente não se encontram abordagens ou modelos sistemáticos para treinamento de melhoria de processos na literatura. Por exemplo, o IDEAL [McFeeley 96], embora indicando as necessidades de treinamento e cobrindo atividades para definir estratégias de treinamento, não fornece suporte suficiente sobre como estes treinamentos podem ser organizados e realizados. Outros artigos somente relatam experiências especificas [Beekman et al 96] [Varkoi e Mäkinen 99], etc.

Por outro lado, a norma ISO 10015 [ISO 99] propõe um processo genérico de treinamento, que parte das necessidades de melhoria da organização e estabelece um programa de treinamento, que procura atender estas necessidades de conhecimento (figura 1). O processo proposto pela norma define quatro estágios para realização do treinamento: (i) definição das necessidades de treinamento; (ii) planejamento do treinamento; (iii) realização do treinamento; e (iv) avaliação dos resultados do treinamento. 


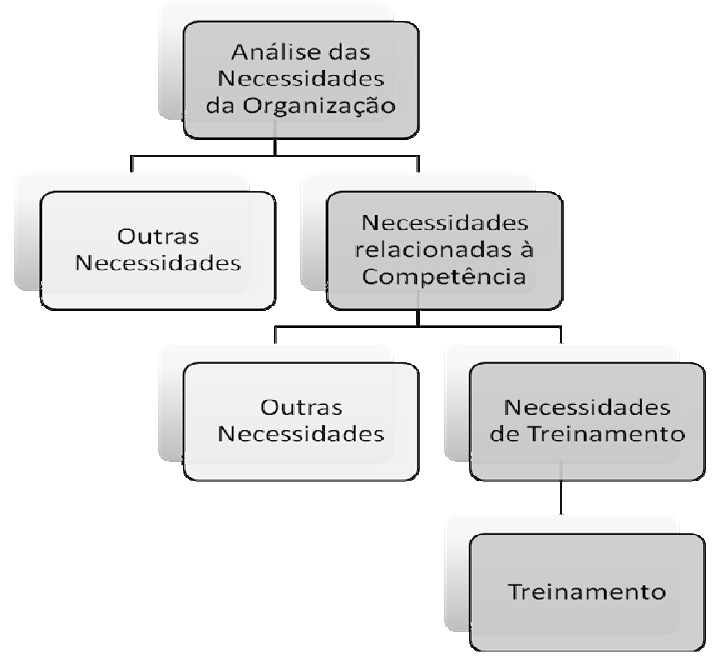

Figura 1. Treinamento para melhoria da qualidade [ISO 99].

A norma ISO 10015, no entanto, oferece uma abordagem genérica e define uma estrutura mínima de fases e atividades para que possa ser estabelecido um programa de treinamentos para a melhoria. Para operacionalizar a norma é necessário sistematizar a sua aplicação. Neste sentido, este artigo apresenta um modelo para programas de treinamento no contexto de programas de melhoria de processo de software.

O artigo é estruturado da seguinte forma: a seção 2 apresenta a abordagem de melhoria de processos ASPEI, seguida pela seção 3 onde o modelo de treinamentos proposto é apresentado em relação ao seu alinhamento à mesma abordagem, incluindo o detalhamento das fases e sessões de treinamento propostas. Na seção 4 são apresentados os resultados observados da aplicação da abordagem em um programa de melhoria e, por fim, a seção 5 apresenta as conclusões do artigo.

\section{A Abordagem ASPEI}

A abordagem ASPEI/MSC (Approach for Software Process Establishment and Improvement in Micro and Small Companies) [Weber 05] [Wangenheim et al. 06] [Hauck 08] foi desenvolvida para suportar o estabelecimento de processos de software de forma incremental em micro e pequenas organizações de desenvolvimento de software. A ASPEI/MSC, além da iniciação, é dividida em quatro fases (figura 2): diagnóstico do processo de software atual, análise estratégica, definição do processo e implantação do processo.

Iniciação: Antes de dar início à melhoria de processo em si, a organização se prepara para o programa de melhoria, estabelecendo as metas da melhoria e a política de qualidade, indicando os papéis e as pessoas serão direta ou diretamente envolvidas no programa de melhoria. É planejado o programa de melhoria, gerando um plano de melhoria, que será seguido durante todo o programa e servirá para monitorar o seu desempenho.

Diagnóstico do processo: O diagnóstico do processo de software atual da organização é realizado por meio de uma avaliação (gap analysis) em alto nível dos processos, levantando os pontos fortes e fracos, bem como estabelecendo as recomendações de 
melhoria a partir da comparação do processo atual com as melhores práticas presentes nos modelos e normas de melhoria.

Análise estratégica: A partir das recomendações de melhoria e das metas estratégicas da organização os processos a serem modelados são priorizados e definidos quais processos serão abordados no primeiro ciclo de melhoria.

Definição dos processos: Em ciclos de melhoria, cada um dos processos identificados na fase anterior é modelado, refinado e documentado em um guia de processo. As oportunidades de melhoria dos processos são então detalhadamente estudadas e as alternativas de solução são propostas a partir da utilização de guias de referência de processo [Hauck 08] que trazem modelos de atividades, técnicas, ferramentas, templates de apoio à modelagem e melhoria dos processos. Cada processo modelado é experimentado em um projeto piloto controlado de forma a avaliar a aplicabilidade do modelo de processo.

Implantação: A partir das experiências obtidas nos projetos piloto, o processo é institucionalizado em toda a organização. É estabelecido um plano de implantação que será seguido para que o processo atinja toda a organização e forneça feedback para melhoria contínua.

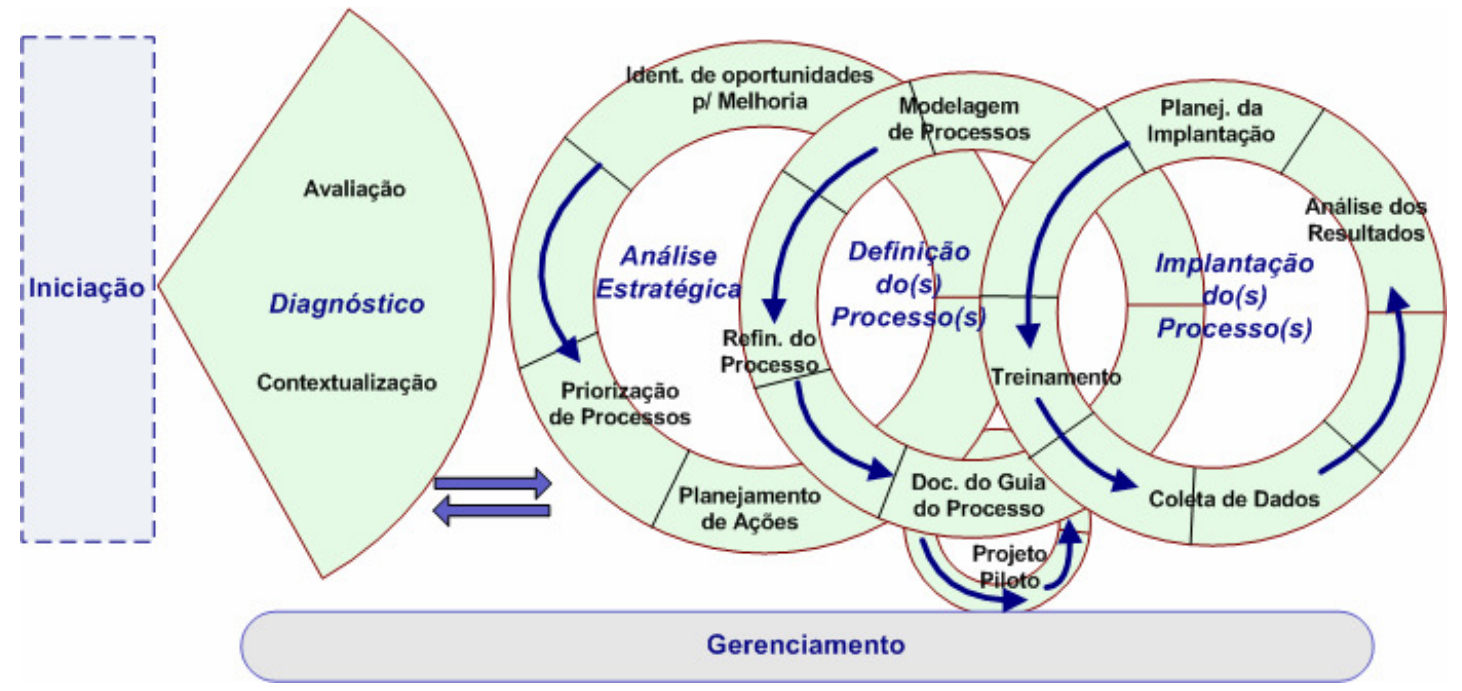

Figura 2. A abordagem ASPEI [Hauck 08].

A abordagem ASPEI/MSC tem sido aplicada com sucesso para estabelecer melhoria de processos em organizações de desenvolvimento de software [Hauck 08] [Hauck 07] [Wangenheim 06] [Weber 05] [Hauck 04] gerando resultados positivos. Nestas aplicações práticas da ASPEI, foram percebidas oportunidades de melhoria no que diz respeito ao programa de treinamentos que apóia a aplicação da abordagem, de forma a estar devidamente alinhado às metas organizacionais de melhoria de processos e às melhores práticas de treinamento relatadas na literatura. A seção seguinte apresenta um modelo de programas de treinamento que procura atender a estes objetivos.

\section{O Modelo de Treinamento}

Partindo das citadas experiências de aplicação da abordagem ASPEI em diversos programas de melhoria e também com base na literatura [Fowler 90] [McFeeley 96] 
[O'Toole 04] [Wiegers 96] [ISO 99], este artigo apresenta um modelo de treinamentos no contexto de programas de melhoria de processo de software.

$\mathrm{O}$ modelo proposto foi concebido com o objetivo de atender às necessidades típicas de programas de melhoria de processo de software, considerando as características específicas deste tipo de iniciativa de forma integrada à abordagem ASPEI/MSC. Estendendo o trabalho apresentado em [Wangenheim et al 08], o modelo de treinamento foi alinhado à norma ISO 10015, atendendo às principais recomendações, artefatos e atividades descritas nesta norma além de detalhar as definições de cada uma das sessões de treinamento.

O resultado é um modelo de programas de treinamento (figura 3) constituído de um conjunto de sessões de treinamento com diferentes objetivos de aprendizagem, audiência e metodologias de ensino, sendo distribuídas durante todo o programa de melhoria de processo.

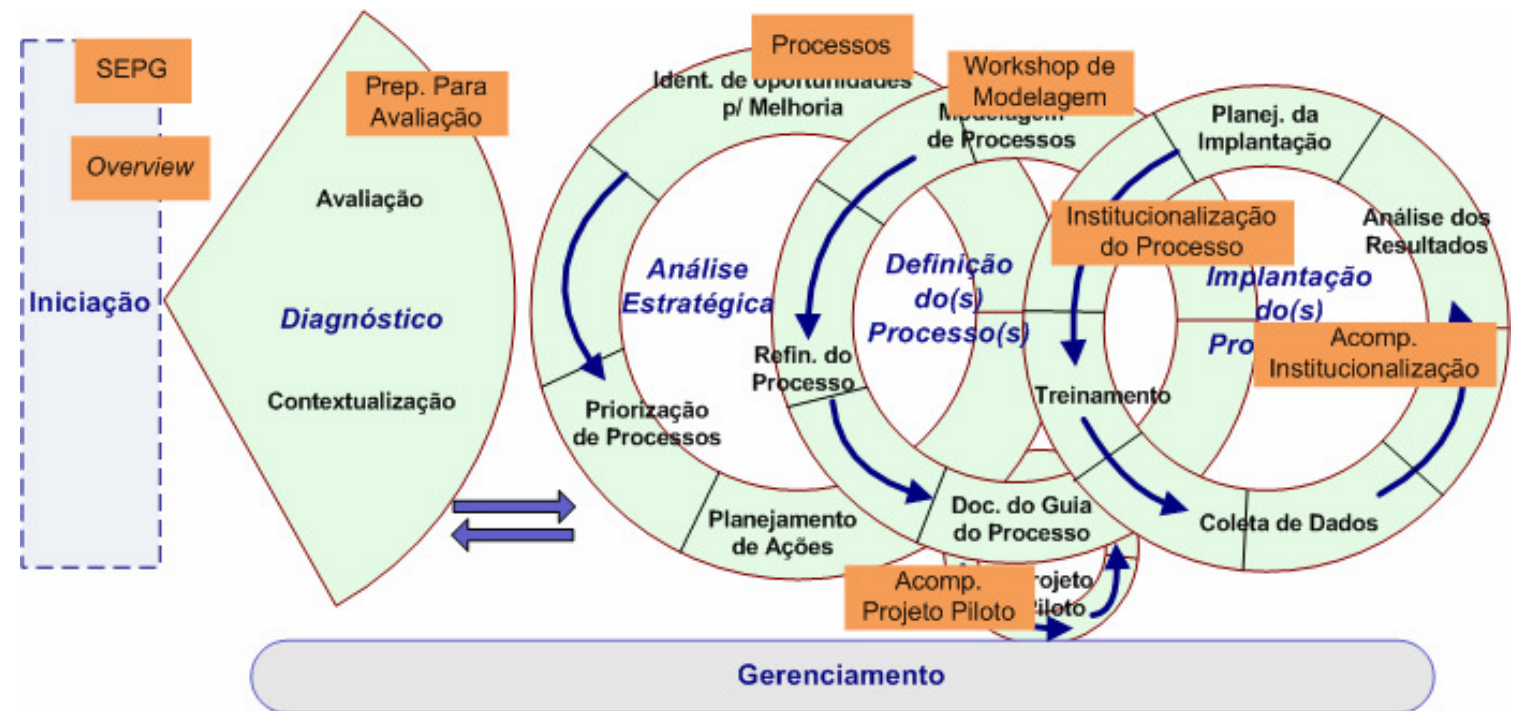

Figura 3. Visão geral do modelo de treinamento na ASPEI/MSC

Algumas sessões de treinamento do modelo são pré-definidas a partir das necessidades de treinamento típicas de programas de melhoria realizadas em alinhamento às ASPEI/MSC. Outras sessões de treinamento são definidas de acordo com as necessidades específicas detectadas pela fase de diagnóstico do processo de software da ASPEI/MSC. Alinhado à norma ISO 10015, o modelo de programa de treinamentos é composto de quatro fases:

1. Definição das necessidades de treinamento: A partir das recomendações de melhoria e do diagnóstico do processo de software, são estabelecidas as necessidades de treinamento da organização. Inicialmente, algumas necessidades básicas devem ser contempladas até que se tenha o diagnóstico da organização que é fornecido somente após a primeira fase da ASPEI/MSC. Após o estabelecimento do diagnóstico, somente os treinamentos alinhados aos objetivos estratégicos e às necessidades da organização serão planejados e executados.

2. Planejamento do treinamento: Um planejamento inicial de treinamentos é realizado juntamente com o plano de melhoria. Neste plano constam os 
treinamentos básicos alinhados à abordagem ASPEI/MSC, tais como: treinamento do SEPG e treinamento de overview. Após a fase de diagnóstico ocorre o refinamento do plano de treinamentos, onde são detalhadamente planejados os treinamentos específicos para a organização, com base nas necessidades de treinamento estabelecidas.

3. Realização do treinamento: as sessões de treinamento são então realizadas de acordo com o planejado e são coletados dados sobre os resultados dos treinamentos.

4. Avaliação dos resultados do treinamento: com base nos resultados coletados dos treinamentos, são realizadas interpretações que geram um feedback sobre a performance dos treinamentos, permitindo avaliar a qualidade dos mesmos em relação ao instrutor, ao material didático, aos recursos utilizados, etc.

Durante o programa de melhoria de processos, as quatro fases de treinamento vão sendo realizadas de forma iterativa, acompanhando os ciclos de melhoria da abordagem ASPEI/MSC. Cada ciclo de melhoria contém diferentes necessidades de treinamento que vão sendo contempladas por sessões de treinamento específicas (figura $3)$.

\subsection{Sessões de treinamento}

Conforme já comentado, no modelo de treinamento proposto algumas sessões são fixas e se referem a atividades de melhoria de processo de software, criando, por exemplo, uma compreensão sobre o que é melhoria de processo, quais são os passos de uma avaliação de processo, etc. Estas sessões de treinamento são alinhadas à teoria de melhoria de processo de software e especificamente à abordagem ASPEI/MSC. Outras sessões de treinamento já mais direcionadas a um processo e às necessidades e características de uma organização especifica necessitam ser customizadas para cada organização.

Nesta seção são apresentadas as sessões de treinamento definidas em relação ao programa de melhoria de processos de software e ao alinhamento à abordagem de melhoria de processos ASPEI/MSC. Cada sessão é definida em termos de:

- Público alvo: participantes do programa de melhoria de processo, ou dos processos que serão objeto da melhoria, que serão capacitados.

- Objetivo de Aprendizagem: objetivos pedagógicos a serem atingidos com o treinamento. Servirão de base para a avaliação dos resultados do treinamento.

- Método de Ensino: Indicação dos métodos que serão utilizados no treinamento, podendo incluir [ISO 99]: expositivo, prático, coaching, à distância, selftraining, etc.

- Duração: Duração proposta para o treinamento. Pode variar em função de outros fatores, como tamanho do grupo ou método de treinamento a ser aplicado.

- Conteúdo: Visão geral dos assuntos que devem ser abordados no treinamento.

As sessões de treinamento realizadas no modelo são:

Treinamento do SEPG: O primeiro treinamento do programa consiste na capacitação dos membros do SEPG (Software Engineering Process Group) da organização. Os 
membros do SEPG da organização são capacitados nos conceitos básicos de melhoria de processos de software e nos fundamentos do(s) modelo(s) de referência de processo que serão utilizados no programa de melhoria. Pode ser substituído por um curso de introdução do modelo de referência (por exemplo: curso introdutório do MPS.BR [SOFTEX 07]):

* Público Alvo: Membros do grupo de Engenharia de Software da organização.

* Objetivo de Aprendizagem: Conhecer e compreender conceitos e processos de melhoria de processo de software e de modelo(s) de referencia.

* Método de Ensino: Aula expositiva / prática.

* Duração: 16 horas

* Conteúdo: Visão geral dos conceitos referentes à melhoria de processo de software e do(s) modelo(s) de referência adotado (s).

Treinamento Overview: Provê um entendimento básico de melhoria de processos e da abordagem de melhoria utilizada (no caso, a ASPEI/MSC). Tem também o objetivo de informar do início da melhoria de processo e motivar os participantes para a importância dos esforços de melhoria.

* Público Alvo: Representantes de todos os setores da organização. Dependendo da disponibilidade, podem participar todos os membros da organização que serão direta ou indiretamente envolvidos na melhoria.

* Objetivo de Aprendizagem: Conhecer conceitos básicos de melhoria de processo de software e conhecer custos e benefícios relacionados.

* Método de Ensino: Aula expositiva.

* Duração: 1 hora

* Conteúdo: Visão geral sobre melhoria de processos.

Preparação para a Avaliação: Proporciona um entendimento básico dos objetivos da avaliação, do processo de avaliação e dos modelos de referência que serão utilizados. Define o papel de cada participante da avaliação do processo.

* Público Alvo: Membros da organização que participarão da avaliação do processo.

* Objetivo de Aprendizagem: Conhecer conceitos e processos de avaliação de processo de software

* Método de Ensino: Aula expositiva e dialogada.

* Duração: 0,5 horas

* Conteúdo: Processo de avaliação

Treinamento de Processos: São realizados os treinamentos básicos específicos de cada processo. Os processos para os quais serão realizados os treinamentos são identificados a partir das carências da organização levantadas na avaliação dos processos, a partir das recomendações de melhoria.

* Público Alvo: Participantes dos processos-alvo da melhoria.

* Objetivo de Aprendizagem: Conhecer e compreender conceitos, processos e técnicas relacionados à área de processo específico.

* Método de Ensino: Aula expositiva. 
* Duração: 16 horas (pode variar dependendo da avaliação das recomendações de melhoria)

* Conteúdo: Conceitos, técnicas e ferramentas do processo

Workshop de Modelagem de Processos: Rápida apresentação dos conceitos envolvidos na modelagem de processos, incluindo a notação utilizada [Thiry et al. 06]. Também são apresentados os objetivos da modelagem e do processo que será modelado.

* Público Alvo: Membros do grupo de Engenharia de Software da organização.

* Objetivo de Aprendizagem: Conhecer a técnica de modelagem de processos.

* Método de Ensino: Aula expositiva e dialogada.

* Duração: 0,25 horas

* Conteúdo: Técnica de Modelagem de Processos adotada.

Acompanhamento do projeto piloto: Acompanhamento da execução do processo nos projetos piloto. O foco é estimular a adoção do processo que foi modelado, propiciando um contato constante dos participantes do processo com o SEPG da organização.

- Público Alvo: Participantes dos projetos piloto.

* Objetivo de Aprendizagem: Conhecer e compreender o processo padrão da organização e aplicá-lo no projeto piloto.

* Método de Ensino: Coaching

* Duração: 2 a 4 horas por semana durante o projeto piloto

* Conteúdo: Processo padrão organizacional.

Institucionalização do Processo: Em preparação à institucionalização dos processos modelados para toda a organização, são realizadas sessões de treinamento para todos os membros, organizados em grupos de públicos-alvo de acordo com os processos treinados. É importante que as sessões de treinamento não sejam longas e utilizem recursos multimídia para auxiliar na motivação para a utilização dos processos modelados.

* Público Alvo: Todos os membros da organização.

* Objetivo de Aprendizagem: Conhecer, compreender e aplicar cada área de processo conforme definido no processo padrão da organização

* Método de Ensino: Aula expositiva com recursos multimídia

* Duração: 2 horas por processo

* Conteúdo: Modelo de processo da organização.

Acompanhamento da institucionalização do processo: Acompanhamento da execução dos processos associada a auditorias internas de alinhamento aos processos.

* Público Alvo: Membros do grupo de Engenharia de Software da organização.

* Objetivo de Aprendizagem: Reforçar o conhecimento, compreensão e aplicação da área de processo conforme o processo padrão da organização.

* Método de Ensino: Coaching.

* Duração: 2 a 4 horas por semana

* Conteúdo: Processo padrão organizacional. 


\subsection{Avaliação do treinamento}

No intuito de verificar se os objetivos organizacionais e de treinamento foram atingidos [ISO 99] são realizadas avaliações após cada sessão de treinamento. Estas avaliações são realizadas com base nos objetivos de aprendizagem definidos para a sessão de treinamento.

Uma primeira avaliação do treinamento é realizada logo após a sessão de treinamento, onde cada participante responde a um questionário de avaliação. $\mathrm{O}$ conteúdo do questionário de avaliação varia de acordo com a sessão de treinamento realizada, entretanto ele tem sempre três grupos de perguntas que devem ser respondidas pelos participantes:

- Conteúdo: O participante é convidado a avaliar o conteúdo ministrado no treinamento, em relação à adequação do conteúdo e os conhecimentos que foram adquiridos durante o treinamento;

- Didática: São avaliadas as questões relativas aos materiais didáticos e recursos utilizados no treinamento;

- Instrutor: Procura-se obter a opinião do participante do treinamento sobre a clareza, objetividade, métodos e técnicas de ensino e conhecimento da matéria do instrutor.

A figura 4 apresenta o extrato de uma ficha de avaliação de treinamento.

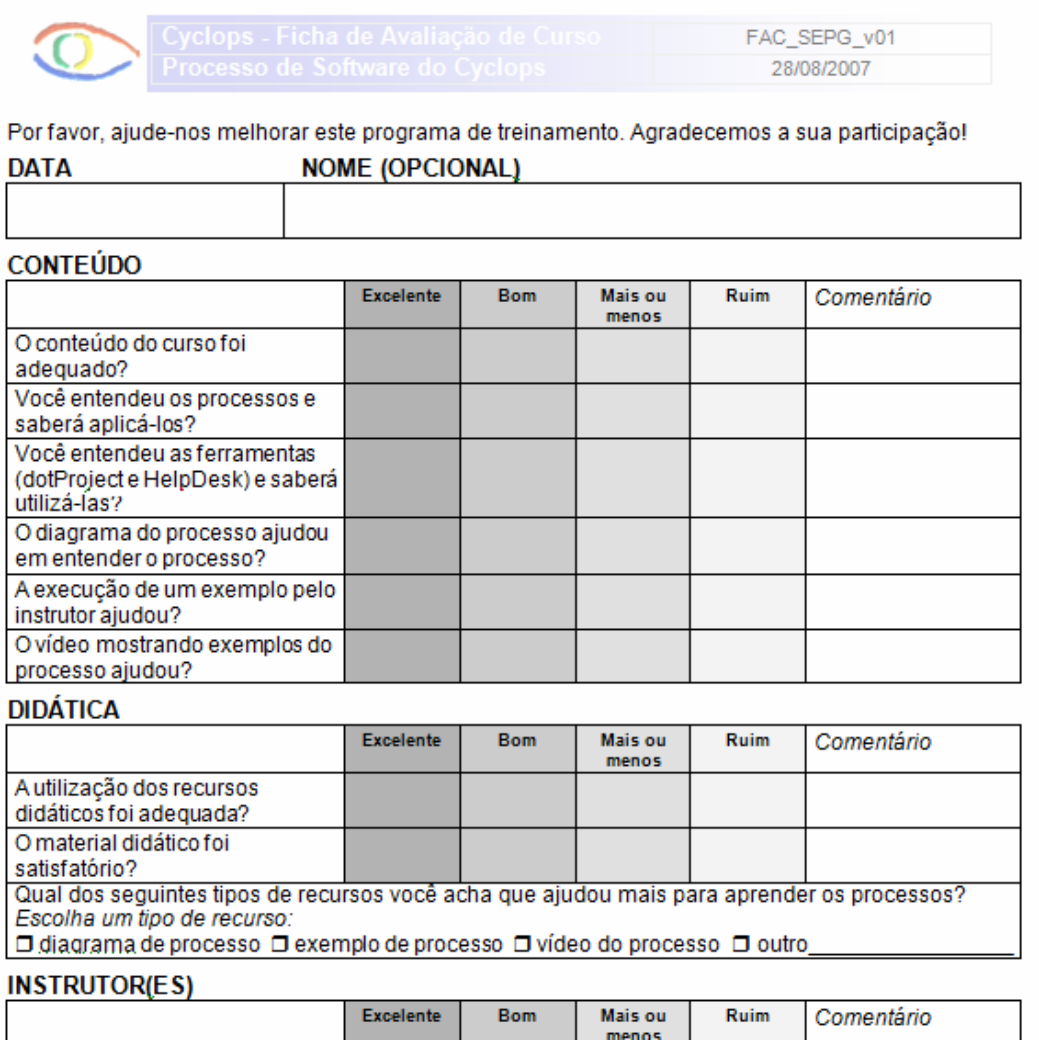

Figura 4: Extrato de ficha de avaliação de treinamento.

Por melhores que sejam as avaliações após a realização das sessões de treinamento, o real efeito dos treinamentos somente será percebido quando os 
participantes do treinamento necessitarem aplicar em situações práticas os conhecimentos adquiridos. Desta forma, os resultados das auditorias de qualidade, ou verificações de alinhamento ao modelo de processo, realizadas nos projetos após os treinamentos, servem para que sejam obtidas avaliações consistentes sobre os resultados práticos dos treinamentos. Os dados de alinhamento da execução dos processos ao processo padrão da organização podem servir como avaliação dos resultados do treinamento, na medida em que sejam cruzados com os objetivos de cada um dos treinamentos realizados.

Os resultados obtidos, tanto dos questionários de avaliação, quanto das auditorias de qualidade, devem ser tratados e reunidos em um relatório [ISO 99]. Este relatório deve ser apresentado durante as reuniões de monitoramento do programa de melhoria, onde serão realizadas análises dos dados coletados e tomadas decisões sobre melhorias a serem realizadas para os próximos treinamentos.

\section{Aplicação do Programa de Treinamentos}

O modelo de treinamentos foi aplicado no grupo de pesquisas CYCLOPS [CYCLOPS 08] da Universidade Federal de Santa Catarina (UFSC). O CYCLOPS é especializado na pesquisa e desenvolvimento de tecnologias inovadoras na área de processamento de imagens médicas, abrangendo mais de cinqüenta pesquisadores em vários laboratórios da UFSC. Esta aplicação foi realizada no contexto de um programa de melhoria guiado pela abordagem ASPEI/MSC focado no nível G do MPS.BR. Na seqüência são relatadas as experiências obtidas em cada uma das sessões de treinamento previstas no modelo proposto.

Como primeiro passo, foi realizado o treinamento dos membros do SEPG, que no grupo CYCLOPS é composto por dois consultores júnior de melhoria de processo. Ambos têm formação em ciência da computação e já possuíam prévios conhecimentos teóricos e práticos sobre SPI e sobre modelos de referência em geral (MPS.BR, CMMI e ISO/IEC 15504) provenientes de programas de melhoria anteriores. Como seria adotado o MPS.BR como modelo de referência pra a melhoria, ambos participaram do curso oficial de introdução ao MPS.BR (16 horas).

Iniciando o programa de melhoria, foi realizado um treinamento de overview, com duração de uma hora para todos os membros do grupo CYCLOPS (incluindo: gerentes de projeto, desenvolvedores, analistas, projetistas, etc.). $\mathrm{O}$ treinamento foi aberto pela coordenação do grupo de pesquisas que promoveu o apoio às iniciativas de melhoria e procurou motivar o grupo para este fim. Em seguida um consultor sênior externo apresentou o plano de melhoria e uma visão em alto nível do MPS.BR.

Em preparação para a avaliação do processo atual, um treinamento curto $(0,5$ horas) foi realizado para nivelar o conhecimento sobre os objetivos da avaliação e o método de avaliação que seria utilizado. O consultor sênior externo deixou claras as questões de confidencialidade e qual papel cada participante da avaliação iria executar, bem como as informações que deveria fornecer ao avaliador.

Com base nas recomendações de melhoria obtidas na avaliação e na priorização dos processos para melhoria, iniciaram-se os treinamentos específicos de cada processo, seguindo a ordem de implementação definida. Foram então realizados treinamentos básicos sobre os conceitos, terminologia, métodos e técnicas de cada um 
dos processos. Estes treinamentos foram percebidos como muito importantes para a modelagem dos processos, que se seguiu. Os treinamentos foram ministrados por consultores sênior, externos ao grupo de pesquisas, apresentando conteúdo e exemplos adaptados à realidade do grupo CYCLOPS. Foram realizados treinamentos de: (i) planejamento de projetos (16 horas); (ii) monitoração e controle de projetos (16 horas), (iii) desenvolvimento de requisitos (32 horas), e (iv) gerência de requisitos ( 8 horas).

Para modelar os processos do CYCLOPS foram realizados workshops de modelagem de processo [Thiry et al. 06]. Em preparação a estes workshops foram realizadas pequenas sessões de treinamento de workshop de processo para os participantes, onde foi apresentada a notação que seria utilizada e como seria realizada a modelagem de processos. Estes treinamentos, realizados para cada grupo que iria modelar o processo, antes do início dos workshops, teve a duração média de 15 minutos cada.

Assim, que um determinado processo estava modelado, seguindo o cronograma definido no plano de melhoria, foram iniciados os projetos piloto. Neste momento foi estabelecido um acompanhamento contínuo à equipe do projeto piloto na forma de coaching (de 2 a 8 horas por semana). Os membros do SEPG visitaram o ambiente de trabalho dos projetos piloto duas vezes por semana e acompanharam a execução das tarefas, sugerindo melhorias e tirando dúvidas dos participantes quanto ao seguimento do processo. Também foram obtidos importantes feedbacks sobre o processo modelado, que serviram de base para melhorias posteriormente implantadas no processo antes da sua institucionalização em toda a organização.

Um dos principais pontos positivos observados no programa de treinamentos é o acompanhamento contínuo. Por melhor que tenha sido o entendimento dos conceitos envolvidos nos processos a serem implantados, é somente durante a sua execução que são realmente percebidas as dúvidas e detectados os pontos onde o modelo de processo precisa ser mais bem detalhado, ou até muitas vezes, alterado, para se adequar à realidade da organização.

No momento em que os processos organizacionais foram definidos e validados nos projetos piloto, para cada um destes processos organizacionais foi realizado um treinamento de Institucionalização do Processo (2 horas cada). Todos os membros da organização, direta ou indiretamente ligados ao processo (por exemplo: planejamento de projetos), participaram dos treinamentos. Estes treinamentos sobre o processo organizacional foram ministrados pelos membros do SEPG do CYCLOPS, apresentando exemplos reais dos projetos do grupo para ilustrar e motivar os participantes ao seguimento do processo. Foram realizadas duas sessões de treinamento para cada processo, com conteúdo idêntico, de forma a possibilitar a participação de todos os membros da organização. Para ilustrar a execução do processo foram produzidos vídeos onde foram apresentados o processo atual e o novo processo definido, incluindo, de forma bem humorada, falhas do processo informal utilizado atualmente. A figura 4 mostra uma cena de um dos vídeos produzidos e utilizados nos treinamentos. Os vídeos têm, no máximo, cinco minutos de duração cada. 


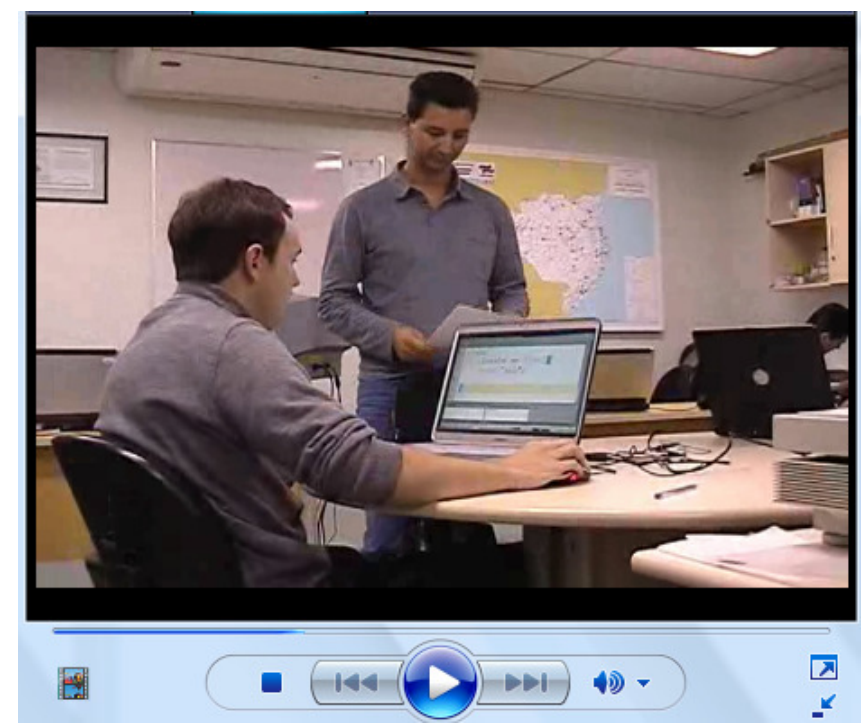

Figura 4. Amostra de vídeo utilizado nos treinamentos

Os vídeos utilizados nos treinamentos foram produzidos pelos membros do SEPG, em um dos laboratórios do grupo de pesquisas. Os atores dos vídeos foram voluntários dentre os gerentes, desenvolvedores e analistas mais diretamente envolvidos nos projetos piloto ou na modelagem do novo processo. Foram apresentados nos vídeos pequenos casos ilustrativos de situações típicas da vida real, recorrentes no processo atual e como elas são resolvidas no novo processo modelado. Os vídeos foram produzidos com recursos mínimos dentro de um período de 4 horas gastando no total aproximadamente 40 homens/hora.

$\mathrm{Na}$ avaliação das sessões de treinamento de institucionalização do processo, o ponto forte mais observado pelos participantes foi a utilização dos vídeos. O uso de situações reais do cotidiano, sendo representadas por colegas do grupo, apresenta o novo processo padrão da organização, de forma atrativa e divertida, ao mesmo tempo em que aponta as principais diferenças e soluções propostas pelo novo modelo.

Outro fator que deve ser ressaltado como uma lição aprendida da realização deste tipo de treinamento refere-se à importância do envolvimento dos membros do SEPG. Em todas as fases de treinamento a cooperação do SEPG junto aos consultores externos na customização dos treinamentos padrões à realidade da organização leva ao melhor aproveitamento dos treinamentos por parte dos membros da organização. Ainda mais importante é este envolvimento quando da realização do treinamento de institucionalização do processo, onde se pôde observar que a apresentação do novo processo organizacional pelo próprio SEPG aos demais membros do CYCLOPS contribuiu fortemente para criar uma atmosfera positiva e motivacional na organização em relação à posterior adoção e coaching.

Após a realização dos treinamentos, os processos modelados passaram a ser executados por toda a organização. Para apoiar a institucionalização foi então iniciado o acompanhamento da institucionalização do processo, realizado na forma de coaching. As sessões de coaching foram realizadas periodicamente durante as auditorias internas de processo, que foram iniciadas paralelamente, de forma a avaliar $\mathrm{o}$ alinhamento da execução dos projetos aos processos modelados. Nestas auditorias a aderência dos projetos ao processo é avaliada, resultando, normalmente, em uma lista de 
não conformidades e num indicador numérico de aderência ao processo padrão para o projeto avaliado. Em paralelo, para cada não conformidade identificada, ações corretivas foram sendo propostas e executadas com base nas recomendações dos membros do SEPG. O coaching é aplicado nas discussões e recomendações geradas durante a definição das ações corretivas. As ações propostas são resultado do acompanhamento da execução das atividades, onde são detalhados aspectos do processo e colhidos feedbacks que auxiliam em ajustes no modelo de processo da organização. No início da institucionalização, foram aplicados vinte homens/hora por semana do SEPG para realização deste acompanhamento de institucionalização. Atualmente este esforço é de dois homens/hora por semana, em média, sendo superior somente quando há alguma solicitação direta de acompanhamento por algum gerente de projeto.

\section{Conclusão}

Este artigo descreve a proposta de um modelo de treinamento para programas de melhoria de processo de software, sua aplicação prática e os primeiros resultados obtidos de sua aplicação. Os principais benefícios observados na utilização do modelo de treinamentos proposto se referem: à customização dos treinamentos ao contexto específico da organização; distribuição dos treinamentos durante todo programa de melhoria; realização dos treinamentos em cooperação com o SEPG e o acompanhamento constante durante os projetos piloto e a institucionalização do processo padrão organizacional.

Embora tendo sido aplicado neste primeiro momento somente em uma unidade de desenvolvimento de software universitária, acredita-se que a transferência do modelo apresentado pode ser realizada para MPEs de software de forma integrada ao próprio programa de melhoria não significativamente impactando em aumento do esforço total aplicado em treinamentos em uma organização. Considerando treinamento em projetos cooperados, o modelo apresentado precisará ser adaptado, dando possibilidades de realizar algumas das sessões de treinamento em conjunto, mas requerendo outras de forma individual por organização.

Como trabalhos futuros, planejamos mais aplicações deste modelo de treinamentos em outros programas de melhoria, além de realizar uma análise comparativa dos resultados obtidos sem a aplicação do modelo.

\section{Agradecimentos}

Agradecemos aos membros do grupo CYCLOPS pelo empenho na realização dos treinamentos e aos consultores da Incremental Tecnologia Ltda. envolvidos na implementação do programa de melhoria no grupo CYCLOPS. Agradecemos também ao Conselho Nacional de Desenvolvimento Científico e Tecnológico - CNPq - Brasil pelo apoio financeiro.

\section{Referências}

Beekman, K. Phelan, J. Rugg, D. (1996) "CMM-Based Software Process Improvement Training: The First Two Years". Conference on $9^{\text {th }}$ Software Engineering Education. 
CMMI Product Team. (2006) "CMMI for Development (CMMI-DEV), Version 1.2". Technical Report CMU/SEI-2006-TR-008, Carnegie Mellon University/ Software Engineering Institute, Pittsburgh, August.

CYCLOPS. (2008) Disponível em: <www.cyclops .ufsc.br>, Acesso em: 01/12/2008.

Daft R. L. (1992) “Organisation Theory and Design”, 4th ed., West Publishing, 1992.

Fowler, P., Rifkin S. (1990) "Software Engineering Process Group Guide". Technical Report CMU/SEI-90-TR-024, September.

Hauck, J. C. R. (2004) "Modelando o Processo de Software em uma Pequena Empresa O Caso VOID CAZ”. SIMPROS - Simpósio Internacional de Melhoria de Processo de Software, São Paulo, 2004.

, J. C. R.; Wangenheim, C. G. v., Thiry, M. (2007) "Suportando a Modelagem de Processo de Monitoração e Controle em Micro e Pequenas Empresas, alinhado ao CMMI, MPS.BR e ISO/IEC15504”. SBQS - Simpósio Brasileiro de Qualidade de Software, Ipojuca - Porto de Galinhas.

J. C. R., Wangenheim C. G. v., Souza R. H., Thiry M. (2008) "Process Reference Guides - Support for Improving Software Processes in Alignment with Reference Models and Standards". Springer - Communications in Computer and Information Science. , v.16, p.70 - 81,

ISO/IEC Std. 15504 (2006) "Information Technology - Process Assessment, Part 1 to Part 5". International Organization for Standardization, 1998-2006.

ISO Std. 10015 (1999) “Quality management - Guidelines for training. International”. Organization for Standardization.

Johnson, D. L. Brodman, J. G. (1999) “Tailoring the CMM for Small Businesses, Small Organizations, and Small Projects, Elements of Software Process Assessment and Improvement", K. El Emam and N.H. Madhavji, eds., IEEE CS Press, pp. 239-259.

McCahon. C. S., Rys, M. J., Ward, K. H. (1996) "The impact of training technique on the difficulty of quality improvement problem solving". Industrial Management \& Data Systems, Vol. 96 No. 7.

McFeeley, R. (1996) "IDEAL: A User's Guide for Software Process Improvement. Handbook" CMU/SEI-96-HB-001, Software Engineering Institute/Carnegie Mellon University, Pittsburgh.

MCT - Ministério da Ciência e Tecnologia. (2005) "Qualidade e Produtividade no Setor de Software Brasileiro, Resultados da Pesquisa 2005" (resultados antecipados recebidos por e-mail do $\mathrm{MCT}$ ).

O'Toole, P. (2004) “Do's and Don'ts of Process Improvement”. SEPG Conference, Orlando. March.

Pressman, R.S. (1996) "Software Process Impediment". IEEE Software, Volume 13, Issue 5, September, pp. $16-17$.

Richardson, I. Wangenheim, C. G. v. (2007) "Why Are Small Software Organizations Different?", IEEE Software, vol. 24, no. 1, pp. 18-22. 
SEBRAE-SP. (2000) "Pesquisa e Planejamento Estratégico. Relatório de Pesquisa: MPEs de Base Tecnológica: Conceituação, formas de financiamento e análise de casos brasileiros", São Paulo.

SEI - Software Engineering Institute. (2006) “Capability Model Integration, version 1.2 - CMMI® for Development, Version 1.2.

SOFTEX. (2007) "Melhoria de Processo de Software Brasileiro - MPS.BR - Guia Geral". Disponível em <http://www.softex.br/mpsbr>. Acesso em 01/12/2008.

STOREY D. J. (1982) “Entrepreneurship and the New Firm”, Croom Helm.

Thiry, M., Wangenheim, C. G. v., Zoucas, A., Pickler, K. (2006) "Uma Abordagem para a Modelagem Colaborativa de Processos de Software em Micro e Pequenas Empresas", V Simpósio Brasileiro de Qualidade de Software - SBQS, Porto Alegre.

Thiry, M., Wangenheim, C. G. v., Zoucas, A., Pickler, K. (2007) "Rumo ao CMMI Nível 2 para Micro, Pequenas e Médias Organizações". In: Instituto Euvaldo Lodi (IEL/SC). (Org.). PLATIC. 1 ed. v. 1, p. 49-79.

Varkoi, T., Mäkinen, T. (1999) "Software Process Improvement Network in the Satakunta Region - SataSPIN". EuroSPI - European Conference on Software Process Improvement, Finland.

Wiegers, K. E. (1996) "Software Process Improvement: Ten Traps to Avoid”. Software Development, May.

Wangenheim, C. G. v., Anacleto A., Salviano C. (2006) "Helping Small Companies Assess Software Processes". IEEE Software, Vol. 23, No. 1, Jan/Feb.

C. G. v., Weber S., Hauck, J. C. R. Trentin G. (2006) "Experiences on Establishing Software Processes in Small Companies". Information and Software Technology, v. 48, n. 9.

, C. G. v., Hauck J. C., Souza R. H., Cancian M. H. (2008) "Experiences in Training for Software Process Improvement" In: Anais da Conference on European Systems \& Software Process Improvement and Innovation - EuroSPI 2008, Dublin.

Weber S. (2005) "ASPE / MSC: Uma Abordagem para Estabelecimento de Processos de Software em Micro e Pequenas Empresas", Dissertação de Mestrado, Universidade Federal de Santa Catarina.

S., Wangenheim C. G. v., Hauck J. C. R. (2005) "Estabelecendo Processos de Software em Micro e Pequenas Empresas", Simpósio Brasileiro de Qualidade de Software, Porto Alegre. 